\title{
The Effects of the Lockdown during the COVID-19 Pandemic on Alcohol and Tobacco Consumption Behavior in Germany
}

\author{
Anne Koopmann $^{\mathrm{a}, \mathrm{b}} \quad$ Ekaterini Georgiadou $^{\mathrm{c}} \quad$ Iris Reinhard $^{\mathrm{d}} \quad$ Astrid Müller $^{\mathrm{e}}$ \\ Tagrid Lemenager ${ }^{a, b}$ Falk Kiefer ${ }^{a, b}$ Thomas Hillemacher ${ }^{c, f}$ \\ ${ }^{a}$ Department of Addictive Behavior and Addiction Medicine, Central Institute of Mental Health, Medical Faculty \\ Mannheim/Heidelberg University, Mannheim, Germany; ${ }^{b}$ Feuerlein Center on Translational Addiction Medicine \\ (FCTS), University of Heidelberg, Heidelberg, Germany; ' Department of Psychiatry and Psychotherapy, Paracelsus \\ Medical University Nuremberg, Nuremberg, Germany; ${ }^{\text {d} D e p a r t m e n t ~ o f ~ B i o s t a t i s t i c s, ~ C e n t r a l ~ I n s t i t u t e ~ o f ~ M e n t a l ~}$ \\ Health, Medical Faculty Mannheim/Heidelberg University, Mannheim, Germany; ${ }^{\text {eDepartment }}$ of Psychosomatic \\ Medicine and Psychotherapy, Hannover Medical School, Hannover, Germany; fDepartment of Psychiatry, Social \\ psychiatry and Psychotherapy, Hannover Medical School, Hannover, Germany
}

\section{Keywords}

Alcohol · Tobacco · Pandemic

\begin{abstract}
Background: Similar to other countries, the government of Germany has implemented various restrictions of social life in March 2020 to slow the spread of COVID-19 pandemic. This results in millions of people being isolated for long periods, which may increase feelings of worry and anxiety. As the consumption of alcohol and tobacco is an often used dysfunctional strategy to cope with such feelings, these restrictions might cause an increase of consumption. Already at the beginning of the COVID-19 pandemic, the World Health Organization (WHO) warned that increased alcohol consumption during the lockdown can increase the prevalence of alcohol use disorders in the future. However, up to now little is known about the changes in alcohol-drinking behavior and tobacco smoking in the general population during the COVID-19 pandemic. Methods: To address this theme, we investigated the changes in alcohol and tobacco consumption in the German population aged between 18
\end{abstract}

and 80 years via an online survey. Results: In total, 3,245 persons participated in the survey; $35.5 \%$ of them reported an increase in drinking during the lockdown $(42.9 \%$ did not change their drinking behavior, $21.3 \%$ drank less, and $0.3 \%$ started drinking). The odds of consuming more alcohol during lockdown were associated with middle age, higher subjective stress due to the COVID-19 pandemic, a lower agreement with the importance of the restrictions, and consuming alcohol more than once per week before the lockdown. Also, $45.8 \%$ of the participants increased their smoking during the lockdown. The odds of smoking more during lockdown were associated with higher subjective stress due to the COVID-19 pandemic. Conclusion: These findings suggest that it is important to start campaigns to inform the general population about potential long-term effects of increased alcohol and tobacco consumption and to raise the health-care professionals' awareness of this topic.

(C) 2021 S. Karger AG, Basel

Falk Kiefer and Thomas Hillemacher shared last authorship.
Correspondence to:

Anne Koopmann, anne.koopmann@zi-mannheim.de 


\section{Introduction}

The new coronavirus disease 2019 (SARS-CoV-2) was first seen at the end of 2019 in Wuhan, China. Its long incubation period and the highly contagious nature of the virus, as well as the extensive global networking with high travel activities all over the world have led to a rapid pandemic spread of SARS-CoV-2. According to Parmet and Sinha [1], the utility of quarantines and travel bans seems to be limited for highly transmissible diseases; however, they are often the first response against new infectious diseases such as the SARS-CoV-2 virus. Like in the majority of countries across the world, the German government has implemented various restrictions on social life and contacts leading to a quarantine-like status to slow the spread of COVID-19 pandemic in all of the sixteen partly sovereign federal states with a difference of a few days, the latest starting at March 23. These restrictions include closures of schools, kindergartens and nonsystemically relevant facilities (e.g., restaurants, cafes, and hairdressing services), travel bans and border closures, cancellation of sporting and cultural events as well as reduction or even permission of social contacts. This so-called lockdown results in millions of people being isolated for long periods, which may subsequently increase feelings of worry and anxiety in the general population.

In a review, Brooks et al. [2] report the impact of quarantine during SARS, EBOLA, and H1N1 influenza pandemic on mental health. In this context, quarantine was defined as restrictions for people who have potentially been exposed to the specific virus and could be contagious. Most of the reviewed studies report psychological distress after quarantine, such as posttraumatic stress symptoms, and some studies even report long-term effects, for example, vigilant handwashing and avoidance of crowds. Only one study assessed the effects of quarantine and social isolation on alcohol-drinking behavior [3]. The authors investigated alcohol abuse and dependence symptoms among 549 employees of a hospital in Beijing, China, that had been affected by the SARS outbreak 2003. They found that the number of fulfilled alcohol abuse/ dependence diagnosis criteria 3 years after the outbreak was positively associated with having been quarantined or having worked in high-risk locations such as SARS wards, during the outbreak. This relationship remained significant even after controlling for sociodemographic and other factors.

Although the current restrictions due the COVID-19 pandemic are not completely equal to quarantine, they represent a quarantine-like status due to their sternness.
Restrictions are set by the government and not voluntary through the people themselves. Thus, this changes peoples' daily life routines and may let them feel socially isolated, which in turn has an impact on their mental health. As the consumption of alcohol and/or tobacco could be (dysfunctional) strategies to cope with such feelings, the restrictions could result in an increase of alcohol and/or tobacco consumption in the general population.

Already at the beginning of the worldwide lockdown, the World Health Organization (WHO) had warned the general public of the potential risks of increased alcohol consumption during social isolation [4]. Due to working in home office, people have less social obligations and there is less control by social surroundings during the lockdown. Based on our clinical experience, this "social control" might have been one reason for reducing or controlling alcohol-drinking behavior to a low level before the lockdown. Focusing on tobacco consumption behavior, the same mechanisms might cause an increase in smoking during the lockdown. According to a systematic review, the odds of a COVID-19 case becoming more severe and leading to death are higher among people with a history of smoking [5]. Therefore, the government of South Africa, where very high prevalence rates of tobacco product use and COVID-19 cases are detected, prohibited the sale of tobacco and nicotine products during the lockdown to curb the spread of the virus and protect vulnerable citizens [6].

Due to the addictive potential of alcohol and tobacco in the long term, excessive misuse of both substances during the isolation caused by the worldwide lockdown might escalate into a substance use disorder in some individuals $[7,8]$. Additionally, neuroadaptation processes in stress and reward pathways during chronically increased alcohol consumption in combination with social stress and feelings of anxiety might cause higher rates of aggression so that there is also a risk for (long-term) consequences for the social surroundings [9]. Like alcohol consumption, cigarette smoking also affects both smokers and those exposed to secondhand smoke [10]. During the lockdown, family members and other persons in direct proximity of tobacco users might be at greater risk for exposure to secondhand smoke if the consumers increase their cigarette smoking [6].

Based on representative data of total revenue in alcoholic beverage in the general population, a significant increase by $6.1 \%$ compared to the mean of corresponding weeks in 2019 was detected [11]. Unfortunately, corresponding representative data of the changes of total revenue in tobacco products in the general population for 
the same period are not available. However, it remained unclear, whether the changes in total revenue in alcoholic beverage were due to stockpiling or reflected real changes in alcohol-drinking behavior during the COVID-19 pandemic.

Based on earlier results of our group on the association between anxiety, stress, and alcohol use $[12,13]$ and data of research concerning the changes in alcohol- and tobacco-consuming behavior in the general population during quarantine or lockdown situations, we built up the hypothesis that especially people experiencing feelings of anxiety and stress due to the COVID-19 pandemic might increase their alcohol and/or tobacco-consuming behavior as a (dysfunctional) skill to cope with these feelings during the lockdown. To address this question, we investigated the changes in alcohol-drinking and tobaccosmoking behavior as well as attitudes and feelings concerning COVID-19 pandemic in the German population during the time of governmental restrictions via an online survey.

\section{Methods}

\section{Recruitment of Participants}

To assess changes in alcohol-drinking and tobacco-smoking behavior during the lockdown, an online survey was created using SoSci Survey version 2.5.00-i (SoSci Survey GmbH, Munich, Germany) (for the questionnaire, see online suppl. material; see www. karger.com/doi/10.1159/000515438 for all online suppl. material). In this survey, we also assessed data concerning the changes in participants' gaming, gambling, eating, and buying behavior that are not the focus of the present paper (data will be reported elsewhere). It was promoted via print and social media canals as well as radio interviews and was posted from April 8 to May 11, 2020. During this period, various restrictions on social life and contacts leading to a quarantine-like status in all of the sixteen partly sovereign federal states in Germany. These restrictions include closures of schools, kindergartens and nonsystemically relevant facilities (e.g., restaurants, cafes, and hairdressing services), travel bans and border closures, cancellation of sporting and cultural events as well as reduction or even permission of social contacts. People aged between 18 and 80 years were invited to participate in the survey. The cover page of the survey included information on what the survey is about, the reason for conducting it, how the data will be used, and the anonymous nature of the survey in accordance to the principles of the Declaration of Helsinki and the EU General Data Protection Regulation. The study protocol was approved by the Ethics Committee of the University of Heidelberg (registration number: 2020-552N; DRKS-ID: DRKS00022268).

\section{Assessment Instrument}

The survey was developed in the Department of Psychiatry and Psychotherapy at the Paracelsus Medical University Nuremberg, Nuremberg General Hospital, and the Department of Addictive
Behavior and Addiction Therapy at the Central Institute of Mental Health Mannheim, Germany. It included categorical assessments of sociodemographic variables, changes in alcohol consumption during the lockdown as well as the frequency of alcohol consumption before the lockdown, and changes in cigarette smoking during the lockdown as well as the amount of cigarette consumption before the lockdown (for the questionnaire, see online suppl. material).

Further questions referred to attitudes and feelings concerning COVID-19 pandemic. Participants were asked to answer questions about their attitudes about the successful controlling of the coronavirus ( $1=$ for sure not, $2=$ probably not, 3 = probably yes, and $4=$ yes, for sure), the importance of the restrictions for the successful control of the coronavirus ( $1=$ for sure not, 2 = probably not, 3 = probably yes, and $4=$ yes, for sure), and the estimated duration of the lockdown (in weeks). Additional questions referred to fear for their own health or for the health of those close to them $(0=$ not at all, $11=$ very much $)$ and perceived stress due to the restrictions during lockdown $(0=$ not at all, $11=$ very much $)$.

\section{Data Analyses}

Statistical analyses were conducted using the IBM SPSS statistical package, version 26.0 (IBM Corporation, Armonk, NY, USA) and SAS/STAT software, version 9.4 of the SAS System for Windows. To characterize the study sample concerning its sociodemographic data as well as feelings and attitudes concerning COVID-19 pandemic, the following descriptive statistics were computed: means, standard deviations, ranges, and frequencies. Based on the information about alcohol and tobacco consumption before and during the lockdown, we built groups "group less," "group same," and "group more" to characterize changes in alcohol- and tobacco-consuming behavior.

For group differences, categorical variables were compared using $\chi^{2}$ tests or exact $\chi^{2}$ tests where appropriate. Additionally, effect size Cramer's V was reported. To explore significant associations between the groups (built based on the changes in alcohol respective tobacco-consumption behavior) and the categorical variables further, we performed post hoc tests with Bonferroni correction and calculated standardized residuals for the cells of the crosstabs which quantify the standardized difference between observed and expected (from the marginal distributions) numbers. These expected counts are the number of cases expected in each cell if the 2 variables were statistically independent.

To explore differences between groups for the variables fear, stress and number of weeks for lockdown, we performed ANOVAs and Bonferroni post hoc analyses. To estimate the relationship between sociodemographic variables as well as attitudes and feelings concerning the COVID-19 pandemic and changes in consuming behavior of alcohol or tobacco during the lockdown, we additionally performed binary logistic regression analyses. As dependent variables, we defined consuming more alcohol during the lockdown $(1=$ Yes, $0=$ No) and smoking more during the lockdown $(1=$ Yes, $0=$ No). The variables age (categorical), gender, categorical assessments of attitudes and feelings of the participants, and categorical assessments of the frequency of alcohol consumption before the lockdown were included as covariates in the model. The significance level for all tests was set at $\alpha=0.050$. A BonferroniHolm correction was used to control for multiple testing in post hoc tests in case of significant categorical predictors with more than 2 categories.
Koopmann/Georgiadou/Reinhard/ Müller/Lemenager/Kiefer/Hillemacher 
Table 1. Descriptive variables $(N=3,245)$

\begin{tabular}{|c|c|c|}
\hline \multirow[t]{2}{*}{ Variables } & \multicolumn{2}{|c|}{$(N=3,245)$} \\
\hline & $N$ & $\%^{\mathrm{a}}$ \\
\hline \multicolumn{3}{|l|}{ Age } \\
\hline $18-24$ years & 394 & 12.1 \\
\hline $25-34$ years & 880 & 27.1 \\
\hline $35-44$ years & 687 & 21.2 \\
\hline $45-54$ years & 630 & 19.4 \\
\hline $55-64$ years & 496 & 15.3 \\
\hline 65 years and older & 158 & 4.9 \\
\hline \multicolumn{3}{|l|}{ Gender } \\
\hline Female & 2,074 & 63.9 \\
\hline Male & 1,162 & 35.8 \\
\hline Diverse & 9 & 0.3 \\
\hline \multicolumn{3}{|l|}{ Living situation } \\
\hline Alone & 794 & 24.6 \\
\hline With partner & 1,118 & 34.6 \\
\hline With children & 135 & 4.2 \\
\hline With partner and children & 703 & 21.8 \\
\hline With parents & 211 & 6.5 \\
\hline Other forms & 271 & 8.4 \\
\hline \multicolumn{3}{|l|}{ School, years } \\
\hline$<11$ & 1,010 & 31.4 \\
\hline $11 \leq x \leq 13$ & 755 & 23.5 \\
\hline$>13$ & 1,452 & 45.1 \\
\hline \multicolumn{3}{|l|}{ Having a systemically relevant profession } \\
\hline No & 1,839 & 58.1 \\
\hline Yes & 1,325 & 41.9 \\
\hline Employment status before the lockdown & 1,706 & 52.7 \\
\hline Full time & 741 & 22.9 \\
\hline Part time & 365 & 11.3 \\
\hline School/university/in training & 238 & 7.3 \\
\hline Retiree & 40 & 1.2 \\
\hline Jobless & 66 & 2.0 \\
\hline Housewife/-man/other & 84 & 2.6 \\
\hline \multicolumn{3}{|c|}{ Changes in employment status during the lockdown } \\
\hline No & 1,560 & 54.4 \\
\hline Yes & 1,309 & 45.6 \\
\hline
\end{tabular}

Add-ups may not be equal to total due to rounding and missing

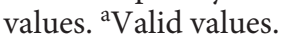

\section{Results}

\section{Demographic Data}

The total sample consisted of $63.9 \%$ females and $35.8 \%$ males, and $0.3 \%$ of the participants reported diverse gender. The majority of the participants reported a high education, with $45.1 \%$ having completed more than 13 school years. More than half of the participants (52.7\%) worked full time before the lockdown, $41.9 \%$ had a systemically relevant profession (e.g., working in the waste management or a hospital or a supermarket), and $45.5 \%$ of the participants had experienced changes in their working situation due to the lockdown (e.g., due to working in home office or due to closure of schools or universities). Demographic data are presented in Table 1.

\section{Attitudes and Feelings Concerning COVID-19 \\ Pandemic}

Information about their attitudes concerning the COVID-19 pandemic and their anxiety for their own health or for the health of those close to them as well as the perceived stress due to restrictions during lockdown is presented in Table 2.

\section{Alcohol Use}

Out of the total sample, 283 participants (8.7\%) did not drink alcohol neither before nor during the lockdown. Out of the remaining 2,962 participants, valid information about changing of drinking behavior during the lockdown is available from 2,896 participants. While 1,243 participants (42.9\%) did not change their drinking behavior during the lockdown, 618 participants $(21.3 \%)$ reported drinking less and 1,027 (35.5\%) reported drinking more during the lockdown. Eight participants (0.3\%) started drinking alcohol during the lockdown.

\section{Group Comparisons}

Participants drinking more ("group more"), drinking less ("group less"), and participants who did not change their drinking behavior ("group same") during the lockdown were collapsed into groups. Table 3 displays the results of group comparisons performed to detect differences between the 3 groups in age (categorical assessment), gender, living situation, school years, profession (systemically relevant profession vs. no systemically relevant profession), employment status before the lockdown, changes in employment status during the lockdown, frequency of alcohol consumption before the lockdown, worries of relatives/friends about participants' alcohol consuming behavior, participants' agreement with the management of the COVID-19 crisis in Germany (dichotomized in yes vs. no), agreement with the importance of the restrictions (dichotomized in yes vs. no), anxiety for the health, and stress due to the exist restrictions.

We found significant differences between the 3 groups for the variables age, living situation, school years, profession, employment status before the lockdown, changes in employment status during the lockdown, frequency of al- 
Table 2. Attitudes and feelings concerning COVID-19 pandemic

\begin{tabular}{|c|c|c|}
\hline Question & Category & Frequency \\
\hline \multirow[t]{3}{*}{ Do you agree that the corona crisis in Germany will be successfully controlled? $\left(n=3,020^{\mathrm{a}}\right)$} & $1=$ for sure not & 192 \\
\hline & $2=$ probably not & 472 \\
\hline & $4=$ yes, for sure & 863 \\
\hline \multirow[t]{3}{*}{ Are the restrictions important for the successful control of the coronavirus? $\left(n=3,139^{\mathrm{a}}\right)$} & $1=$ for sure not & 176 \\
\hline & $2=$ probably not & 299 \\
\hline & Mean (SD) & Range \\
\hline What do you think, how many weeks will the exit restrictions in their current form continue? $\left(n=2,632^{\mathrm{a}}\right)$ & $6.81(8.31)$ & $0-108$ \\
\hline Are you afraid for your health or for the health of those close to you? $\left(n=3,245^{\mathrm{a}}\right)$ & $6.13(2.82)$ & $1-11$ \\
\hline Do you feel stressed by the exit restrictions? $\left(n=3,218^{\mathrm{a}}\right)$ & $5.41(3.29)$ & $1-11$ \\
\hline
\end{tabular}

Add-ups may not be equal to total due to missing values. SD, standard deviation. ${ }^{a}$ Valid values.

cohol consumption before the lockdown, worries of relatives/friends about participants' alcohol consuming behavior, agreement with the importance of the restrictions (dichotomized in yes vs. no), anxiety for the health, and stress due to the exist restrictions.

When looking at post hoc tests and standardized residuals, we observed group differences as described in Table 3. For example, for the category "age" the results of Table 3 can be interpreted as following: it can be seen from Table 3 that respondents' age is significantly related to changes in alcohol consumption during the lockdown $\left(\chi^{2}=156.73 ; \mathrm{df}=10 ; p<0.001\right)$. The superscripts denote that the respondents aged between 18 and 24 years can be found significantly more often in the group drinking less than in both other groups. These participants were also more often found in "group more" than in "group same" but less often in "group more" than in "group less."

When focusing on in-group differences for the abovementioned variables in the 3 different drinking groups, we found the following effects.

\section{Age}

In the group drinking more alcohol ("group more"), participants aged between 35 and 44 years were more often found than expected and less often participants older than 54 years. In the group drinking less alcohol ("group less"), we found more participants aged between 18 and 24 years than expected and less participants aged between 35 and 54 years. In the group drinking the same ("group same"), we found more frequently participants older than
44 years; thus, from this age on participants tend to maintain their alcohol consumption.

\section{Living Situation}

Participants living together with partners and children were less frequently found in "group less," whereas those living with parents were observed more frequently than expected in this group. Participants living together with partner or partner and children were found more often in "group same." Participants living alone or with parents were observed more often in the "group less."

\section{Education}

Participants with lower education were found less often in "group less."

\section{Profession}

Participants having a systemically relevant profession were observed more frequently than expected in "group same" and less frequently in "group more," whereas participants without a systemically relevant profession were observed more often in "group more."

\section{Employment Status}

Part-time employees were observed less frequently than expected in "group less," but more often in "group same." Participants attending school were more often found in "group less" and less frequently in "group same," while participants, who did not work were more often found in "group same" and less often in "group more" than expected. 


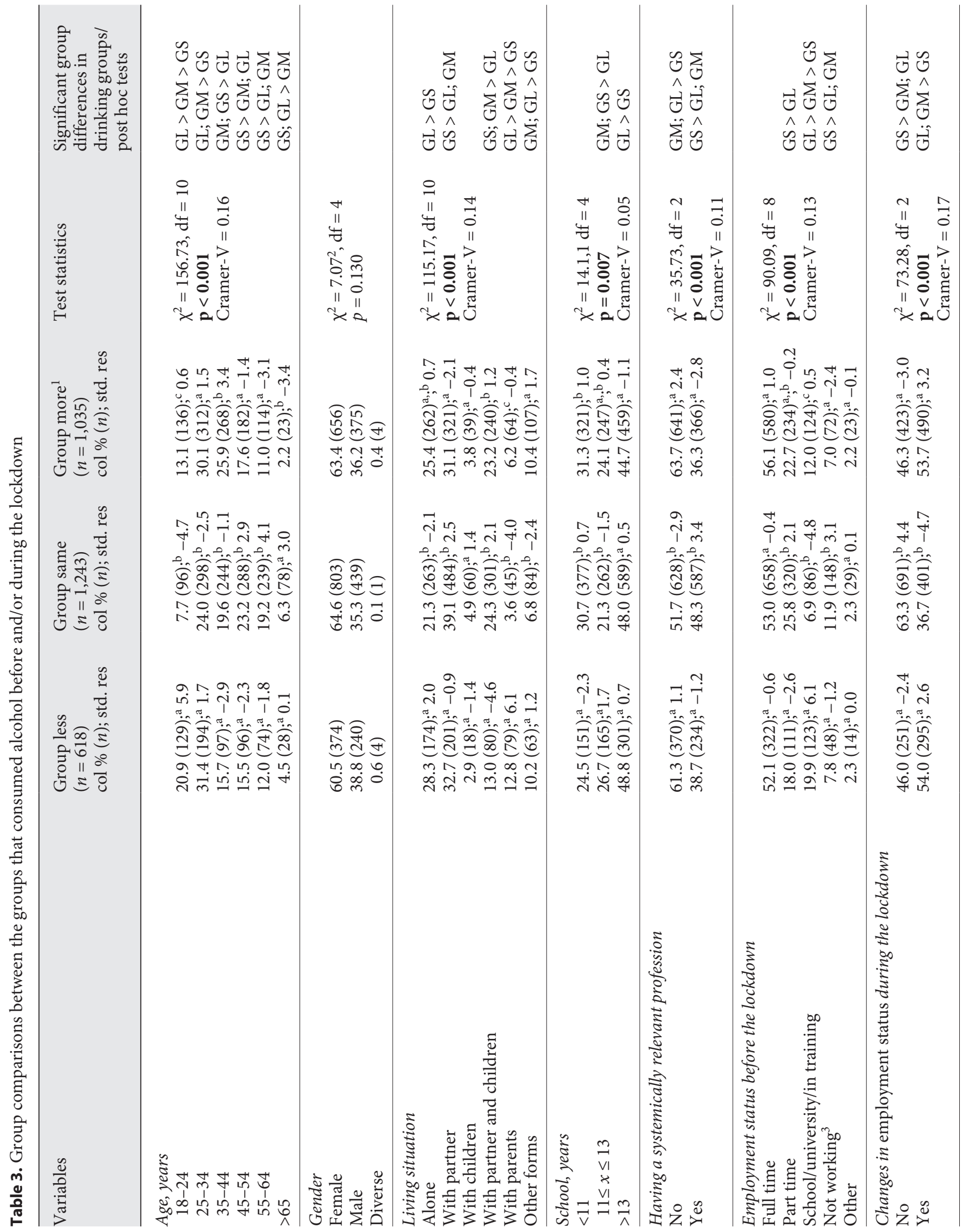




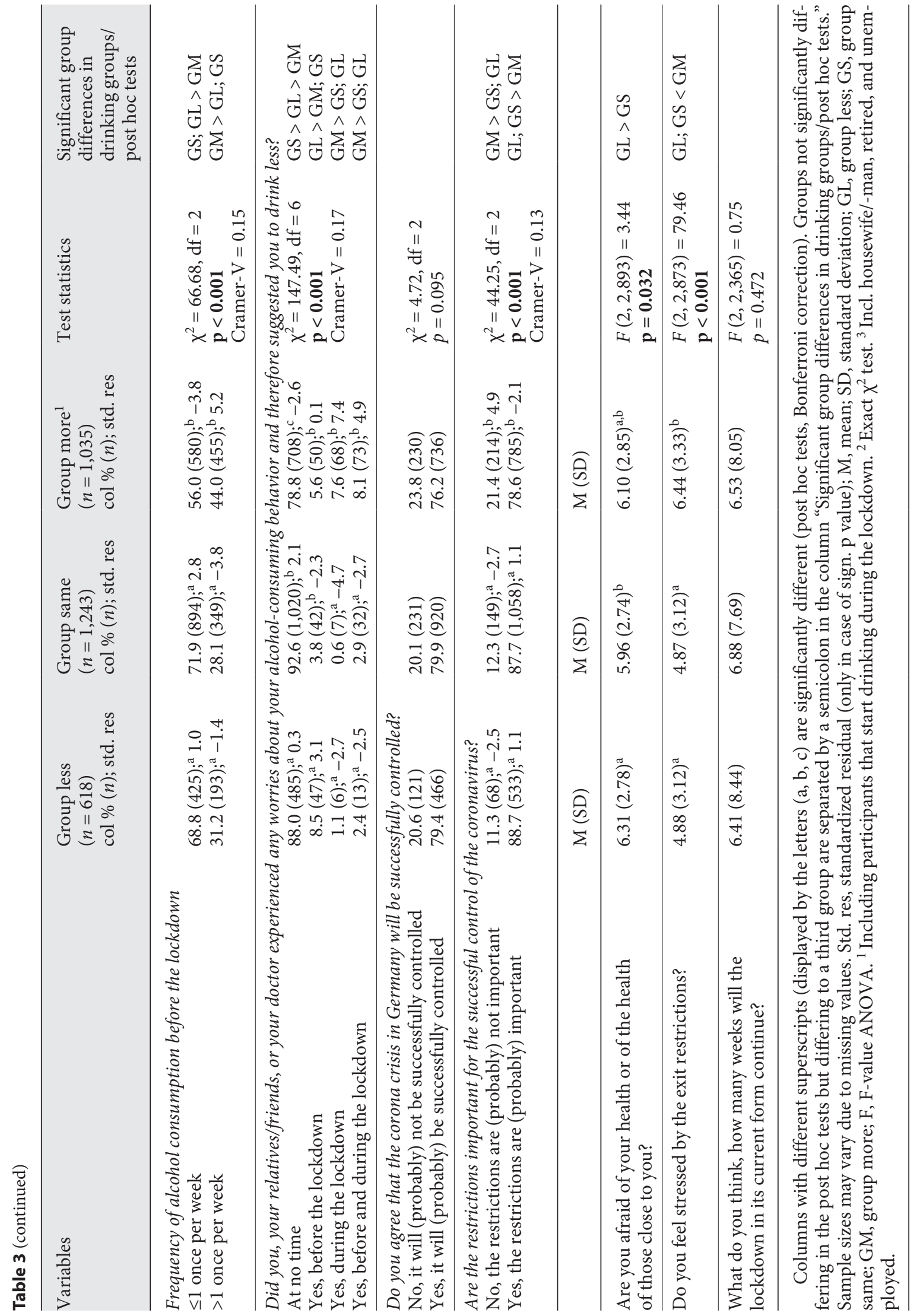


Changes in Employment Status during the Lockdown

Participants who experienced changes in their employment status were more frequently found in the groups "group more" and "group less" but less frequently in "group same," thus changed their consumption a lot, whereas participants without changes did not.

\section{Frequency of Alcohol Consumption before the}

Lockdown

Participants who were drinking more than once per week were found more often than expected in "group more," whereas participants who were drinking once per week or less were observed less often in "group more."

\section{Participants' Agreement with the Importance of the}

Restrictions

Participants who think that the restrictions are (probably) important for the successful control of the COVID-19 pandemic were found less frequently in "group more." Contrarily, participants regarding the restrictions as not so important were more often found in "group more" than expected.

\section{Worries}

Participants with relatives/friends worrying about their alcohol consumption during the lockdown and at both times before and during the lockdown were found more often in "group more," whereas these without having relatives/friends worrying about their alcohol consumption were less often observed in this group.

\section{Feelings about COVID-19 Pandemic}

Considering the variables feelings about COVID-19 pandemic together, there was a significant difference found in analyses of variance between the 3 groups for anxiety $(p=0.032)$ and stress $(p<0.001)$. Additionally, post hoc tests show a significant difference between the groups that drink same and less concerning the pandemic-related health fears with "group less" $(M=6.31)$ showing higher fears than "group same" $(M=5.96)$ and concerning stress levels with "group more" $(\mathrm{M}=6.44)$ reporting higher stress levels than the "group less" $(\mathrm{M}=4.88)$ and "group same" $(\mathrm{M}=4.87)$.

\section{Binary Logistic Regression Analyses}

To estimate the relationship between sociodemographic variables as well as attitudes and feelings concerning the COVID-19 pandemic and changes in alcohol-consuming behavior during the lockdown, we performed binary logistic regression analyses with alcohol consuming behavior as dependent variable $(1=$ consuming more alcohol during the lockdown; $0=$ consuming less alcohol showing no changes in alcohol-consuming behavior during the lockdown). The variables age (categorical assessment with 6 different categories), gender, categorical assessments of attitudes and feelings of the participants, and categorical assessments of the frequency of alcohol consumption before the lockdown (all of them dichotomized) were included as predictors in the model. These binary logistic regression analyses showed that age, stress due to COVID-19 pandemic, the frequency of alcohol consumption before the lockdown, and the agreement with the importance of the restrictions are significant predictors for consuming more alcohol (see Table 4).

\section{Age}

After adjustment for multiple comparisons (Bonferroni-Holm) of the different age-groups, we found a significantly increased risk for consuming more alcohol during the lockdown for the 4 younger groups aged between 18 and 54 years versus the eldest age-group ( $\geq 65$ years) (all $p$ $<0.009$ ), as well as versus the second eldest group (55-64) (all $p<0.030$ ). Furthermore, the 2 groups aged between 25 and 44 years showed an increased risk versus the group aged between 45 and 54 years $(p=0.002$ and $p<0.001)$.

\section{Stress}

Each point on the scale increases the risk for consuming more alcohol during the lockdown by $14 \%$.

\section{Frequency of Alcohol Consumption before the \\ Lockdown}

Participants consuming alcohol more than once per week had a 2.3-fold risk for increasing their alcohol consumption during the lockdown.

\section{Importance}

Participants who found the restrictions (probably) important for successful control have a significantly lower risk for consuming more alcohol during the lockdown.

\section{Nicotine Use}

Out of the total sample, valid information about smoking is available from 3,116 participants. 2,360 (75.7\%) of them did not smoke neither before nor during lockdown, while $756(24.3 \%)$ participants reported to smoke before and/or during the lockdown. Seventy-five (9.9\%) of them quit smoking, and 30 (4.0\%) participants started smoking during the lockdown, while 237 participants (31.3\%) did 
Table 4. Binary regression analyses regarding increased alcohol consumption

\begin{tabular}{|c|c|c|c|}
\hline & OR & $95 \% \mathrm{CI}$ & $p$ value \\
\hline \multicolumn{3}{|l|}{ Age } & $<0.001$ \\
\hline $18-24$ years old & 2.87 & $1.66-4.96$ & $<0.001$ \\
\hline 25-34 years old & 3.03 & $1.80-5.09$ & $<0.001$ \\
\hline $35-44$ years old & 3.53 & $2.09-5.97$ & $<0.001$ \\
\hline $45-54$ years old & 2.04 & $1.20-3.47$ & 0.008 \\
\hline $55-64$ years old & 1.42 & $0.82-2.46$ & 0.206 \\
\hline$\geq 65$ years old & \multicolumn{3}{|l|}{ Reference } \\
\hline \multicolumn{3}{|l|}{ Gender } & 0.442 \\
\hline Female (1) & 1.07 & $0.90-1.28$ & 0.442 \\
\hline Male (2) & \multicolumn{3}{|l|}{ Reference } \\
\hline Anxiety due to COVID-19 pandemic (1-11) & 0.99 & $0.96-1.03$ & 0.681 \\
\hline Stress due to COVID-19 pandemic (1-11) & 1.14 & $1.10-1.17$ & $<0.001$ \\
\hline & $<0.001$ \\
\hline$\leq 1$ time per week & Reference & & \\
\hline & 2.30 & $1.92-2.76$ & $<0.001$ \\
\hline \multicolumn{3}{|c|}{ In your opinion, do you agree that the corona crisis in Germany will be successfully controlled? } & 0.756 \\
\hline No, it will (probably) not be successfully controlled & Reference & & \\
\hline Yes, it will (probably) be successfully controlled & 0.97 & $0.78-1.20$ & 0.756 \\
\hline \multicolumn{3}{|c|}{ In your opinion, are the restrictions important for the successful control of the coronavirus? } & 0.008 \\
\hline No, the restrictions are (probably) not important & Reference & & \\
\hline Yes, the restrictions are (probably) important & 0.70 & $0.54-0.91$ & 0.008 \\
\hline \multicolumn{4}{|l|}{ Model fit statistics } \\
\hline-2 log likelihood & \multicolumn{3}{|l|}{$3,165.5$} \\
\hline Nagelkerke pseudo $R^{2}$ & \multicolumn{3}{|l|}{0.132} \\
\hline
\end{tabular}

not change their smoking behavior, 68 participants (9.0\%) smoked less, and 346 (45.8\%) smoked more during the lockdown.

Participants who smoke less ("group less"), smoke more ("group more"), and did not change their smoking behavior ("group same") during the lockdown were collapsed into groups. Table 5 displays the results of group comparisons performed to detect differences between the 3 groups in age (categorical assessment), gender, living situation, school years, profession (systemically relevant profession vs. no systemically relevant profession), employment status before the lockdown, changes in employment status during the lockdown, amount of cigarette consumption before the lockdown, worries of relatives/ friends about participants' tobacco-consuming behavior, participants' agreement with the management of the COVID-19 crisis in Germany (dichotomized in yes vs. no), agreement with the importance of the restrictions (dichotomized in yes vs. no), anxiety for the health, and stress due to the exist restrictions.
For nicotine-consuming behavior, we found significant differences between the 3 groups for the variables age, living situation, employment status before the lockdown, changes in employment status during the lockdown, worries of relatives/friends about participants' tobacco-consuming behavior, agreement with the successful control and the importance of the restrictions (dichotomized in yes vs. no), and stress due to the exist restrictions.

When looking at post hoc tests and standardized residuals, we observed group differences as described in Table 5. For example, for the category "age" the results of Table 5 can be interpreted as following: it can be seen from Table 5 that the respondents' age is significantly related to changes in nicotine-consuming behavior during the lockdown $\left(\chi^{2}=25.18 ; \mathrm{df}=10 ; p=0.005\right)$. The superscripts denote that the respondents aged between 18 and 24 years can be found significantly more often in the group smoking less than in both other groups. Whereas in "group same" and "group more," the same 
Table 5. Group comparisons between the groups that smoked before and/or during the lockdown

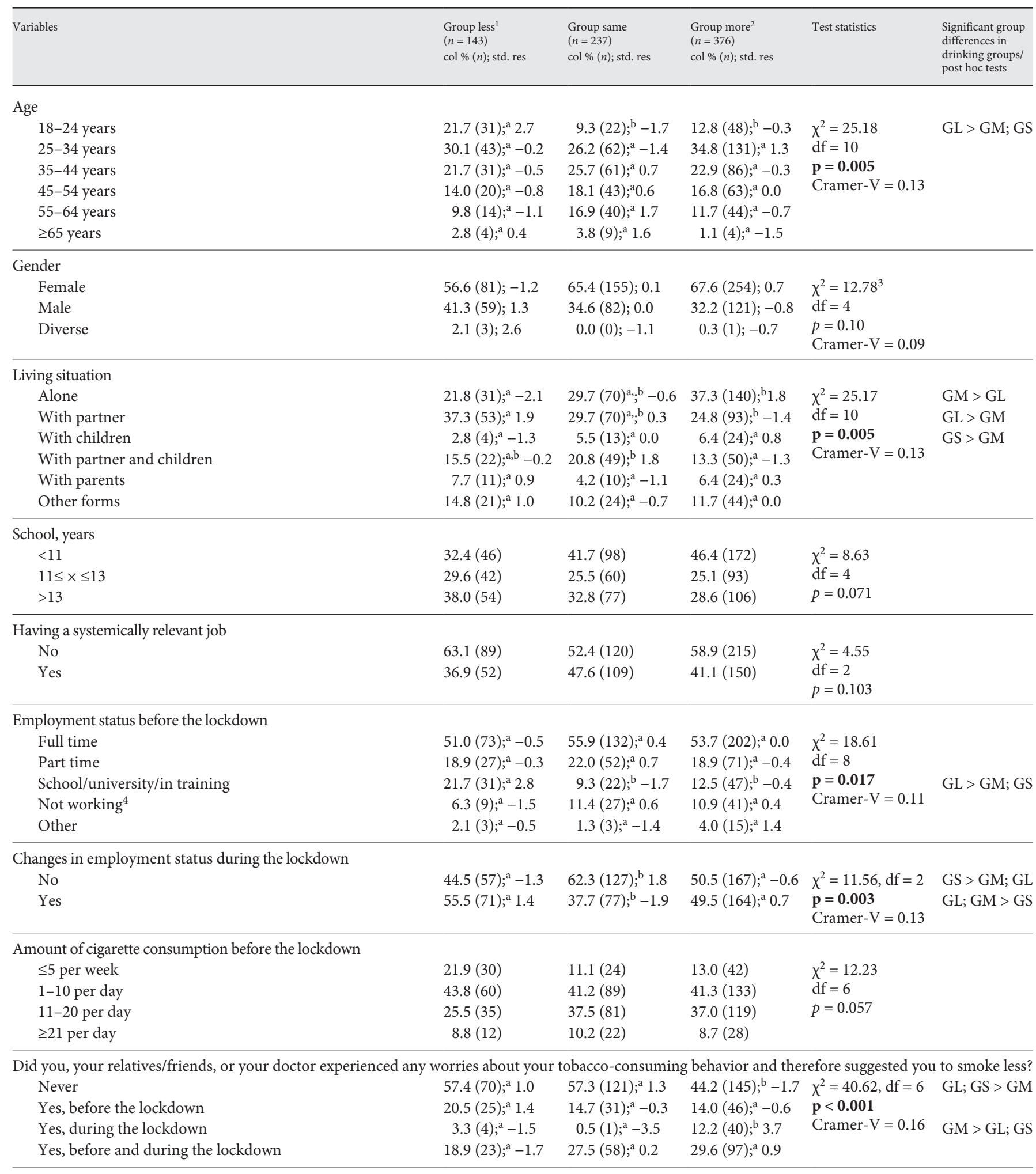


Table 5 (continued)

\begin{tabular}{|c|c|c|c|c|c|}
\hline Variables & $\begin{array}{l}\text { Group less }^{1} \\
(n=143) \\
\text { col \% }(n) \text {; std. res }\end{array}$ & $\begin{array}{l}\text { Group same } \\
(n=237) \\
\text { col \% (n); std. res }\end{array}$ & $\begin{array}{l}\text { Group more }{ }^{2} \\
(n=376) \\
\text { col \% (n); std. res }\end{array}$ & Test statistics & $\begin{array}{l}\text { Significant group } \\
\text { differences in } \\
\text { drinking groups/ } \\
\text { post hoc tests }\end{array}$ \\
\hline \multicolumn{6}{|c|}{ Do you agree that the corona crisis in Germany will be successfully controlled? } \\
\hline No, it will (probably) not be successfully controlled & $21.5(29) ;^{\mathrm{a}}-1.7$ & $28.1(62) ;^{\mathrm{a}, \mathrm{b}}-0.4$ & $33.4(114) ;^{b} 1.4$ & $\chi^{2}=6.94, \mathrm{df}=2$ & $\mathrm{GM}>\mathrm{GL}$ \\
\hline Yes, it will (probably) be successfully controlled & $78.5(106) ;{ }^{\mathrm{a}} 1.1$ & $71.9(159) ; ;^{\mathrm{a}, \mathrm{b}} 0.2$ & $66.6(227) ;^{b}-0.9$ & $\begin{array}{l}\mathbf{p}=\mathbf{0 . 0 3 1} \\
\text { Cramer-V }=0.10\end{array}$ & $\mathrm{GL}>\mathrm{GM}$ \\
\hline \multicolumn{6}{|c|}{ Are the restrictions important for the successful control of the coronavirus? } \\
\hline No, the restrictions are (probably) not important & $17.6(24) ;{ }^{\mathrm{a}, \mathrm{b}}-0.6$ & $10.5(23) ;^{\mathrm{b}}-3.1$ & $26.2(95) ;{ }^{\mathrm{a}} 2.7$ & $\chi^{2}=21.62, \mathrm{df}=2$ & $\mathrm{GM}>\mathrm{GS}$ \\
\hline \multirow[t]{2}{*}{ Yes, the restrictions are (probably) important } & $82.4(112) ; ;^{\mathrm{a}, \mathrm{b}} 0.3$ & $89.5(196) ;^{b} 1.5$ & $73.8(268) ;^{\mathrm{a}}-1.4$ & $\begin{array}{l}\mathbf{p}<\mathbf{0 . 0 0 1} \\
\text { Cramer-V = } 0.17\end{array}$ & $\mathrm{GS}>\mathrm{GM}$ \\
\hline & $\begin{array}{l}\text { Group less } \\
\text { M (SD) }\end{array}$ & $\begin{array}{l}\text { Group same } \\
\text { M (SD) }\end{array}$ & $\begin{array}{l}\text { Group more } \\
\mathrm{M}(\mathrm{SD})\end{array}$ & & \\
\hline $\begin{array}{l}\text { Are you afraid of your health or of the health of those close to } \\
\text { you? }\end{array}$ & $6.25(2.92)$ & $6.30(2.72)$ & $6.33(3.00)$ & $\begin{array}{l}F(2,753)=0.04 \\
p=0.964\end{array}$ & \\
\hline Do you feel stressed by the exit restrictions? & $5.94(3.27)^{\mathrm{a}}$ & $4.94(3.23)^{\mathrm{b}}$ & $6.57(3.40)^{\mathrm{a}}$ & $\begin{array}{l}F(2,745)=17.13 \\
\mathbf{p}<\mathbf{0 . 0 0 1}\end{array}$ & $\mathrm{GS}<\mathrm{GL} ; \mathrm{GM}$ \\
\hline $\begin{array}{l}\text { What do you think, how many weeks will the lockdown in its } \\
\text { current form continue? }\end{array}$ & $6.13(5.63)$ & $6.62(6.42)$ & $7.66(9.47)$ & $\begin{array}{l}F(2,614)=2.01 \\
p=0.136\end{array}$ & \\
\hline
\end{tabular}

Columns with different superscripts (displayed by the letters (a. b, c) are significantly different (post hoc tests, Bonferroni correction). Groups not significantly differing in the post hoc tests but differing to a third group are separated by a semicolon in the column "Significant group differences in drinking groups/post hoc tests." Sample sizes may vary due to missing values. Std. res, Standardized Residual (only in case of sign. $p$ value); M, mean; SD, standard deviation; GL, group less; GS, group same; GM, group more; F, F-value ANOVA. ${ }^{1}$ Including participants who quit smoking during the lockdown. ${ }^{2}$ Including participants who started smoking during the lockdown. ${ }^{3}$ Exact $\chi^{2}$ test. ${ }^{4}$ Incl. housewife/-man, retired, and unemployed.

number of participants aged between 18 and 24 years was found.

When focusing on in-group differences for the abovementioned variables in the 3 different drinking groups, we found the following effects.

\section{Age}

There is a significant association between the changes in smoking behavior and the different age-groups. Young participants were found more often than expected (from the marginal distributions) in "group less" than in "group same" and "group more." After Bonferroni correction, none of these post hoc tests remained significant.

\section{Gender}

The significant effect for this variable is triggered only by the category "divers" and therefore is not discussed further.

\section{Living Situation}

Participants living alone were found less often than expected in "group less," but more frequently in "group more." Living with a partner leads to the opposite result.
Participants living together with a partner and children are found more often in the "group same" than in "group more."

\section{Employment Status}

Participants attending schools/universities were seen more frequently in "group less" than in both other groups.

\section{Changes in Employment Status during the Lockdown}

Participants who did not experience changes in their employment status were more frequently found in "group same" than in the 2 other groups, whereas participants experiencing changes in their employment status were seen less often in "group same" but more often in "group less" and "group more."

\section{Worries}

Participants who or whose relatives/friends never experienced worries about their consuming behavior were seen less often in "group more" than in the remaining 2 groups, whereas participants experiencing worries during the lockdown were found more often in "group more" than in the 2 other groups. 
Table 6. Binary regression analyses regarding increased nicotine consumption

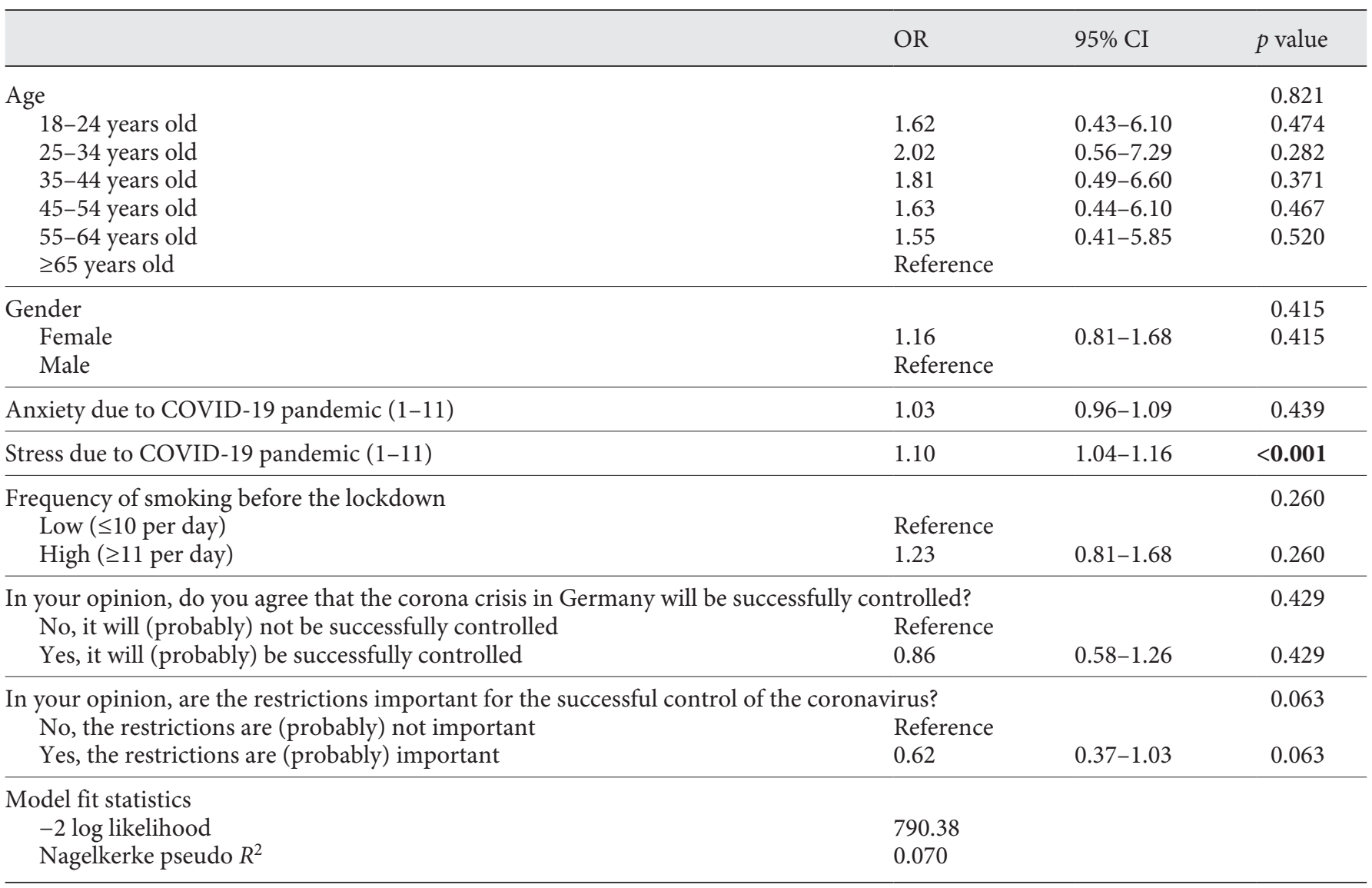

Agreement with the Successful Control of the COVID-19 Crisis in Germany

Participants agreeing with the successful control of the COVID-19 crisis in Germany were found more often in "group less" than in "group more," whereas participants who do not were found more often in the "group more" than in the "group less."

\section{Agreement with the Importance of the Restrictions}

Participants agreeing with the importance were found more often in "group same" than in "group more." Participants who do not agree with the restrictions were seen more often in "group more" than in "group same."

\section{Stress}

Compared to "group less and "group more," participants in "group same" experienced the lowest stress due to the COVID-19 pandemic.

\section{Binary Logistic Regression Analyses}

To estimate the relationship between sociodemographic variables as well as attitudes and feelings concerning the COVID-19 pandemic and changes in tobacco-consuming behavior during the lockdown, we performed binary logistic regression analyses with tobacco-consuming behavior as dependent variable $(1=$ smoking more cigarettes during the lockdown; $0=$ smoking less cigarettes showing no changes in tobacco-consuming behavior during the lockdown).

The variables age (categorical assessment with 6 different categories), gender, categorical assessments of attitudes and feelings of the participants, and categorical assessments of the amount of cigarettes smoked before the lockdown (all of them dichotomized) were included as predictors in the model.

This logistic regression analysis showed that stress due to COVID-19 pandemic is a significant predictor for smoking more with one point on the stress scale increasing the risk for smoking more by $10 \%$. By contrast, 
the other variables did not show a significant effect when tested in a multiple regression model (see Table 6).

\section{Discussion}

The present survey examined the changes in alcoholand tobacco-consuming behavior during the lockdown. The main result of this survey indicates that $35.5 \%$ of the participants drank more (additionally $0.3 \%$ started drinking) and $21.3 \%$ drank less alcohol during the lockdown. Focusing on tobacco-consuming behavior, our results indicate that out of the smoking participants, $45.8 \%$ smoked more (additionally 4.0\% started smoking), 9.0\% smoked less, and $9.9 \%$ of them quit smoking during the lockdown.

On the one hand, these results suggest that the higher total revenue in alcoholic beverage in the general population seen in representative data of the German Federation of Consume Research (GfK) [11] are not due to stockpiling but reflect real changes in alcohol-drinking behavior in parts of the population. On the other hand, they underline the concerns of the WHO toward the potential risks of increased alcohol consumption in the general population during social isolation [4], at least for Germany. Especially younger and middle-aged participants and participants with higher alcohol consumption before the lockdown, as well as those experiencing higher stress due to the COVID-19 pandemic or a lower agreement with the importance of the restrictions, seem to have a higher risk for increasing their alcohol consumption during the lockdown. Additionally, experiencing higher stress due to the COVID-19 pandemic also seems to be a risk factor for increasing tobacco-consuming behavior during the lockdown. By showing this, our results confirm the suggestions of Rehm et al. [14] regarding the impact of past public health and economic crises on alcohol consumption. Here, the authors postulated 2 main scenarios with opposite predictions regarding the impact of the current COVID-19 pandemic on the level and patterns of alcohol consumption. In the first scenario, the authors see an increase in consumption for some parts of the population, due to distress experienced as a result of the pandemic, while in the second they are predicting the opposite outcome, a lowered level of consumption, based on the decreased physical and financial availability of alcohol. In contrast to our results which show that at the same time parts of the population showed an increase of substance consume, while others show a decrease. Rehm et al. [14] predicted a decrease in substance consumption for the immediate future, while up on their opinion the expected increase in consumption in the first scenario may become more relevant in the medium- and longer-term future.

The last result with $9.9 \%$ of the smokers quitting smoking might probably be quite surprising as smoking is often used to cope with psychosocial stress in humans, so we expected an increase in cigarette smoking, but not such a high proportion of participants, who stopped smoking during the lockdown. However, this result seems to be similar with results of an online survey in the Belgian general population during the COVID-19 pandemic, which could show that $6.5 \%$ of the smokers quit smoking during the lockdown [15]. One reason for smoking cessation during lockdown might have been the fact that it is well known that the SARS-CoV-2 virus primarily affects thelung. Therefore, some people might have stopped smoking to reduce their SARS-CoV-2 virus-associated risks.

In comparison to the results of the online survey on alcohol and tobacco consumption in the general population in Belgium during the lockdown [15], there are some similarities as well as some differences to our results. The survey in Belgium was online during the lockdown in this country from April 8 to April 29. The number of participants and their mean age as well as the gender ratio in both countries were very similar (Belgium: 3,632 participants, mean age 42.1 [SD $=14.6$ ], $70 \%$ females; Germany: $3,245$ participants, mean age 41.1 [SD $=14.1], 63.9 \% \mathrm{fe}-$ males). In both countries, about one-third of the participants reported an increase of alcohol consumption during the lockdown. By contrast, in comparison to our results, in the Belgian online survey less smokers took part (24.3\% of the participants in our sample vs. $16.4 \%$ in the Belgian sample). In both samples, age in years seems to be a negative predictor for an increased alcohol during the lockdown. While in Belgium, nonhealth-care workers seem to have increased odds and students a decreased odd for an increased alcohol consumption during the lockdown, we found that working in a not-system-relevant job seems to be a predictor for an increased alcohol consumption during the lockdown. In the Belgian survey, the number of children seems to be a predictor for an increased alcohol consumption; unfortunately, we did not assess this information in our survey. However, we asked the participants about their perceived stress due to COVID-19 pandemic, which showed increased odds for an increased alcohol consumption during the lockdown. Participants' perceived stress could also be due to dealing with closed schools and caring about their children. In contrast to the results concerning the changes in alcohol
Koopmann/Georgiadou/Reinhard/ Müller/Lemenager/Kiefer/Hillemacher 
consumption, the predictors for an increased nicotine use seem to be completely different in the 2 study populations (Belgian survey: age, living situation, educational background, and homework vs. German survey: stress). These results reflect that there seems to be some transnational effects of governmental restrictions on substance consuming behavior in the general population during lockdown. However, some of the effects seem to be country specific. These point out that there is a need of more transnational research to elucidate the effects of the lockdown during COVID-19 pandemic on alcohol and tobacco consumption in Europe.

A higher risk for an increase in alcohol or in tobaccoconsuming behavior and higher levels of perceived stress during the COVID-19 pandemic in some parts of the population might also be due to their lower socioeconomic status (e.g., lower income and constricted living situations) and less-developed supportive social networks. Additionally, chronic alcohol use leads to neuroadaptations in stress and reward pathways, which result in dysfunctional hypothalamic pituitary adrenocortical and sympathetic adrenomedullary axes including dysregulation of the cortisol response and deficits in emotional regulation [16]. In turn, these neuroadaptations can result in an increased craving for alcohol in response to stress [16]. As stated in the introduction, neuroadaptation processes in stress and reward pathways in response to chronically increased alcohol consumption in combination with social stress and feelings of anxiety might result in higher rates of aggression of the consumers and thereby also increase the risk for (long-term) consequences for their social surroundings [9]. It might be hypothesized that some of the smoking participants also increased their tobacco consumption to cope with such psychosocial stress and aggression. Due to the complex living and social situation of the participants with a low socioeconomic status, this group was already a high-risk population for domestic violence before the beginning of the lockdown, and therefore, this situation may be exacerbated during the lockdown.

Although the presented results are based on a high number of participants out of the general population, it must be kept in mind that there are limitations. The sample in our survey is a convenience sample with a relatively high proportion of young well-educated females and the recruiting methods exclude the "offline" population that does not use the internet. Due to this lack of representativeness, results are not applicable for the entire German population. The effect sizes of the significant results are small to moderate. Also, we did not include informa- tion about the situations in that participants consumed alcohol before the lockdown, for example, at home alone or in social situations. Furthermore, the cross-sectional design of our study prevents any causal interpretation. Future longitudinal studies should therefore address both risk as well as protection factors for increased substance use during lockdown situations.

Nevertheless, based on the results of this survey, it seems to be important to inform the general population and especially the high-risk group, perceiving highstress levels during the lockdown, of the risk of an increased substance-consuming behavior during the lockdown and its potential long-term effects and to establish low-threshold medical and social services addressing this problem already during the acute phase of the COVID-19 pandemic. The potential of long-term effects suggests that a quarantine-like status as practiced during the lockdown should be kept as long as necessary but as short as possible. Additionally, there is a need for more research on the changes in consumption behavior of alcohol and/or tobacco during the COVID-19 pandemic and its long-term effects. Last, it seems to be necessary to raise the health-care professional's awareness to look at changes in alcohol and tobacco consuming behavior of their patients and to help them to find specific treatments.

\section{Statement of the Ethics}

The study protocol was approved by the Ethics Committee of the University of Heidelberg (registration number: 2020-552N; DRKS-ID: DRKS00022268). Participants of this survey have given their written informed consent to publish their data. For data collection, we used an anonymous online survey.

\section{Conflict of Interest Statement}

All authors have no conflicts of interest.

\section{Funding Sources}

The study was not funded by a finding source.

\section{Authors Contributions}

A.K., E.G., F.K., and T.H. designed the survey. E.G, I.R., and A.K. analyzed data. A.K. prepared the first draft; all authors provided comments and revised the manuscript. 


\section{References}

1 Parmet WE, Sinha MS. Covid-19: the law and limits of quarantine. N Engl J Med. 2020 Apr 9;382(15):e28.

2 Brooks SK, Webster RK, Smith LE, Woodland L, Wessely S, Greenberg N, et al. The psychological impact of quarantine and how to reduce it: rapid review of the evidence. Lancet. 2020;395(10227):912-20.

3 Wu P, Liu X, Fang Y, Fan B, Fuller CJ, Guan $\mathrm{Z}$, et al. Alcohol abuse/dependence symptoms among hospital employees exposed to a SARS outbreak. Alcohol. 2008;43(6):706-12.

4 World Health Organization (WHO). Available from: http://www.euro.who.int/de/ health-topics/health-emergencies/coronavirus-covid-19/novel-coronavirus-2019-ncovtechnical-guidance/coronavirus-disease-covid-19-outbreak-technical-guidance-europe/ mental-health-and-covid-19.

5 Vardavas C, Nikitara K. COVID-19 and smoking: a systematic review of the evidence. Tob Induc Dis. 2020 Mar 20;18:20.
6 Egbe CO, Ngobese SP. COVID-19 lockdown and the tobacco product ban in South Africa. Tob Induc Dis. 2020 May 6;18:39.

7 Clay JM, Parker MO. Alcohol use and misuse during the COVID-19 pandemic: a potential public health crisis? Lancet Public Health. 2020 May 5;5(5):e259.

8 Ornell F, Moura HF, Scherer JN, Pechansky F, Kessler FHP, von Diemen L. The COVID-19 pandemic and its impact on substance use: implications for prevention and treatment. Psychiatry Res. 2020 May;289:113096.

9 Beck A, Heinz A. Alcohol-related aggressionsocial and neurobiological factors. Dtsch Ärztebl Int. 2013;110(42):711-5.

10 Barnoya J, Glantz SA. Cardiovascular effects of secondhand smoke: nearly as large as smoking. Circulation. 2005;111(20):2684-98.

11 German Federation of Consumer Research (GfK): Consumer Panel FMCG March 2020.

12 Kiefer F, Jahn H, Otte C, Naber D, Wiedemann K. Hypothalamic-pituitary-adrenocortical axis activity: a target of pharmacological anticraving treatment? Biol Psychiatry. 2006; 60(1):74-6.
13 Koopmann A, Leménager T, Wolf ND, Reinhard I, Hermann D, Koch J, et al. The impact of atrial natriuretic peptide on anxiety, stress and craving in patients with alcohol dependence. Alcohol Alcohol. 2014;49(3):282-6.

14 Rehm J, Kilian C, Ferreira-Borges C, Jernigan D, Monteiro M, Parry CDH, et al. Alcohol use in times of the COVID 19: implications for monitoring and policy. Drug Alcohol Rev. 2020 May;39(4):301-4.

15 Vanderbruggen N, Matthys F, Van Laere S, Zeeuws D, Santermans L, Van den Ameele S, et al. Self-reported alcohol, tobacco, and cannabis use during COVID-19 lockdown measures: results from a web-based survey. Eur Addict Res. 2020;26(6):309-15.

16 Koob G, Kreek MJ. Stress, dysregulation of drug reward pathways, and the transition to drug dependence. Am J Psychiatry. 2007; 164(8):1149-59. 